Meta

Journal des traducteurs

Translators' Journal

\title{
Domaines économiques et axes d'embauche des philologues français et des traducteurs en Pologne
}

\section{Anna Kuznik et Virginie Little}

Volume 62, numéro 1, avril 2017

URI : https://id.erudit.org/iderudit/1040467ar

DOI : https://doi.org/10.7202/1040467ar

Aller au sommaire du numéro

\section{Éditeur(s)}

Les Presses de l’Université de Montréal

\section{ISSN}

0026-0452 (imprimé)

1492-1421 (numérique)

Découvrir la revue

Citer cet article

Kuznik, A. \& Little, V. (2017). Domaines économiques et axes d'embauche des philologues français et des traducteurs en Pologne. Meta, 62(1), 69-93. https://doi.org/10.7202/1040467ar
Résumé de l'article

L’objectif de cet article est de déterminer dans quelles conditions s'est opérée l'insertion professionnelle des diplômés des études de philologie française, y compris des traducteurs, détenteurs d'un diplôme de premier cycle (licence) en Pologne de 2011 à 2014. Dans une approche inductive, nous analysons quatre groupes de données empiriques, collectées parmi les étudiants de l'Institut d'études romanes de l'Université de Wrocław et leurs employeurs. Deux groupes de données (deux enquêtes) correspondent au versant « étudiants » et ont été présentés en détail dans une publication précédente. Dans le présent article, nous nous penchons sur les deux groupes de données suivants, provenant cette fois-ci du versant « employeurs » : une analyse du contenu de la documentation élaborée à l'occasion de la gestion des stages professionnels et des offres d'emploi de différents types, adressées à tous les étudiants dudit institut. L'image finale synoptique qui en résulte, comportant une configuration des domaines économiques et des axes d'embauche, représente une situation cohérente, dans laquelle, avec la progression des études, les domaines économiques se concrétisent et s'enrichissent, et les axes d'embauche se dessinent plus nettement. 


\title{
Domaines économiques et axes d'embauche des philologues français et des traducteurs en Pologne
}

\author{
ANNA KUZNIK \\ Uniwersytet Wrocławski, Wrocław, Pologne \\ anna.kuznik@uwr.edu.pl \\ VIRGINIE LITTLE \\ Little Infinity Consulting, Varsovie, Pologne \\ virginie@little-infinity.com
}

\begin{abstract}
RÉSUMÉ
L'objectif de cet article est de déterminer dans quelles conditions s'est opérée l'insertion professionnelle des diplômés des études de philologie française, y compris des traducteurs, détenteurs d'un diplôme de premier cycle (licence) en Pologne de 2011 à 2014. Dans une approche inductive, nous analysons quatre groupes de données empiriques, collectées parmi les étudiants de l'Institut d'études romanes de l'Université de Wrocław et leurs employeurs. Deux groupes de données (deux enquêtes) correspondent au versant «étudiants» et ont été présentés en détail dans une publication précédente. Dans le présent article, nous nous penchons sur les deux groupes de données suivants, provenant cette fois-ci du versant «employeurs »: une analyse du contenu de la documentation élaborée à l'occasion de la gestion des stages professionnels et des offres d'emploi de différents types, adressées à tous les étudiants dudit institut. L'image finale synoptique qui en résulte, comportant une configuration des domaines économiques et des axes d'embauche, représente une situation cohérente, dans laquelle, avec la progression des études, les domaines économiques se concrétisent et s'enrichissent, et les axes d'embauche se dessinent plus nettement.
\end{abstract}

\section{ABSTRACT}

The aim of this paper is to identify the conditions of occupational integration of undergraduate students in French philology, including translators, in Poland between 2011 and 2014. Applying an inductive methodology, we analyse four pools of empirical data, collected from students of the University of Wrocław's Institute of Romance Studies and their employers. The initial two pools of data (two surveys) reflect the students' side and are presented in detail in a previous paper. In the current paper, we deal with the final two pools of data, proper to the employers' side: the content analysis of the documentation generated during the work placement activities and of the job offers received by this Institute. The global image obtained, in terms of areas of economic activity and employability axes, shows a coherent situation: as undergraduate studies progress, the former become more specific and varied, and the latter become more firmly established.

\section{RESUMEN}

En este artículo nos proponemos identificar las condiciones en las cuales se ha producido la inserción laboral de los estudiantes del primer ciclo (licenciatura) de la carrera de filología francesa, incluyendo a los traductores, en Polonia entre los años 2011 y 2014. Siguiendo un planteamiento metodológico inductivo, analizamos cuatro conjuntos de datos empíricos, recogidos del alumnado del Instituto de Estudios Románicos de la Universidad de Wrocław y de sus empleadores. Los dos primeros grupos de datos (dos 
encuestas), que corresponden a la vertiente de los estudiantes, están analizados en detalle en una publicación anterior. En cambio, en el presente artículo nos centramos en la otra vertiente, la de los empleadores. Los datos proceden del contenido de la documención generada durante la gestión de las prácticas profesionales y de las ofertas de trabajo de diferente tipo, dirigidas a todos los estudiantes del Instituto. La imagen sinóptica final que surge de nuestro análisis, con la configuración resultante de las áreas de la actividad económica implicadas y de los ejes de la empleabilidad identificados, hace pensar en una situación coherente en la cual, junto con la progressión de los estudios, las áreas de la actividad económica se van concretando y enriqueciendo, y los ejes de la empleabilidad se van reafirmando.

\section{MOTS CLÉS/KEYWORDS/PALABRAS CLAVE}

domaines économiques, axes d'embauche, formation supérieure, philologie française, traduction

areas of economic activity, axes of employability, higher education, French philology, translation

áreas de la actividad económica, ejes de la empleabilidad, educación superior, filología francesa, traducción

\section{Introduction}

Dans le présent article, nous nous concentrons sur un espace particulier et crucial dans lequel l'offre d'emploi à destination des philologues français formés dans nos universités satisfait la demande d'emploi. Nous analysons le cas des spécialistes de la langue française et de la traduction en Pologne. Nous décrivons les paramètres et les conditions dans lesquels cette rencontre s'opère, en divisant les acteurs impliqués en deux groupes: versant «étudiants» et versant "employeurs», comme deux versants convergents de la même montagne. Nous nous intéressons aux motivations de ces deux groupes d'acteurs unis par le même dialogue et aux domaines économiques concrets qui constituent l'espace de leur rencontre. Sans une motivation pour dialoguer, aucune rencontre fructueuse ne se produit jamais et sans des sujets de conversation, les deux groupes d'acteurs restent muets.

En suivant une approche méthodologique inductive, nous analysons quatre groupes de données empiriques, collectées pendant trois années universitaires (20112012, 2012-2013, 2013-2014) parmi nos étudiants inscrits aux études de philologie française, au premier cycle (licence), à l'Institut d'études romanes (IÉR, Instytut Filologii Romańskiej), à la Faculté des lettres (Wydział Filologiczny) de l'Université de Wrocław (UWr, Uniwersytet Wrocławski) et parmi leurs employeurs réels et potentiels, tous situés au cœur de la région polonaise de la Basse-Silésie (Dolny Śląsk) dans le sud-ouest du pays.

Dans cet article, nous utilisons le terme traditionnel employé en Pologne dans les institutions d'enseignement supérieur, "philologie française» et "philologue français» pour nous référer aux études de langue et civilisation françaises et aux étudiants en langue et civilisation françaises (études de lettres françaises).

Notre étude est une étude de cas: l'échantillon est circonstanciel et la situation décrite peut s'avérer éphémère. Bien que les données empiriques aient été collectées pendant trois années universitaires successives, nous sommes conscientes qu'elles ne servent qu'à illustrer certaines tendances générales, dans une vision dont l'intérêt est 
synchronique et qui reste restreinte à un contexte de formation supérieure précis, ce qui exclut a priori leur capacité à être généralisées à tout un collectif concerné et à expliquer des processus historiques d'une manière diachronique.

Comme chaque étude de cas (Yin 1994; voir Creswell 2003: 179-207; Flyvbjerg 2004; voir aussi Rojo 2013: 53-87), nos données empiriques réunies et les réflexions qu'elles motivent sont fortement liées au contexte économique et éducatif du pays. C'est pourquoi nous y consacrons toute la section 2 du présent article, les conclusions ne pouvant se référer qu'à ces situations, qui gardent certaines similitudes avec le contexte polonais que nous décrivons. Chaque lecteur jugera les similitudes, l'utilité et les applications possibles selon son propre contexte économique et éducatif. De plus, par notre étude, nous avons voulu illustrer et tester un type de démarche méthodologique en plusieurs étapes qui peut être réutilisé par d'autres chercheurs.

Notre analyse s'inspire, du point de vue méthodologique et conceptuel, du travail de Bowker (2004), qui, à partir de la perspective des employeurs, a réuni et étudié 301 offres d'emploi, diffusées pour pourvoir à des postes variés dans le secteur de la traduction (traducteurs, interprètes, terminologues, localisateurs, etc.) parues au Canada de janvier 2000 à décembre 2002. Parmi ces 301 annonces, 78 provenaient du tableau d'annonces dans l'École de traduction et d'interprétation de l'Université d'Ottawa. Les 301 annonces avaient été numérisées et analysées avec le logiciel WordSmith Tools. Les résultats furent comparés à ceux présentés en 1999 par le Comité sectoriel de l'industrie canadienne de la traduction. Le travail de Bowker correspondant à une réalité antérieure de plus de 10 ans à la nôtre et propre à un contexte spécifique, officiellement bilingue (anglais et français), nous n'établissons cependant pas de comparaisons directes entre les résultats de Bowker et les nôtres.

Nous avons présenté le versant «étudiants» le 25 octobre 2014 au cours de la $20^{\mathrm{e}}$ rencontre du réseau thématique «La traduction comme moyen de communication interculturelle», rencontre portant comme titre "Vingt-cinq ans après... Traduire dans une Europe en reconfiguration» (IÉR UWr, Pologne, du 23 au 25 octobre 2014) dans une communication orale intitulée «Le travail pour les philologues français et les traducteurs: axes d'embauche et domaines de l'activité économique», et dans le présent article, le versant "étudiants» est traité d'une manière abrégée (texte publié dans Kuznik 2015). Cette fois-ci, nous déployons l'autre versant, versant « employeurs », complémentaire au premier.

Dans les sections suivantes, le lecteur trouvera une contextualisation de notre étude, le sommaire de notre analyse du versant "étudiants», le détail de notre analyse du versant «employeurs» et les conclusions.

\section{Contextualisation de l'étude}

\subsection{Pologne, Basse-Silésie, Wrocław}

Depuis la fin des années 1990, la Pologne suscite l'intérêt des investisseurs étrangers. Le pays offre en effet aux entreprises étrangères de nombreux avantages. Avec 38 millions d'habitants, elle représente le plus gros marché des pays d'Europe centrale et de l'Est. Depuis 2004 en outre, les investisseurs bénéficient des avantages d'un État membre de l'Union européenne (UE) (et de Scheghen depuis 2007), politiquement stable, qui est une porte d'entrée vers les pays plus à l'est. Généralement bien formés, 
les Polonais font partie des peuples suivant le plus des études supérieures et parlent en général une ou plusieurs langues étrangères. Enfin, les salaires sont encore inférieurs à ceux de l'Europe de l'Ouest - même si cette différence se réduit, voire n'existe plus dans certains secteurs. Le salaire moyen mensuel en 2012 atteignait 890 euros, pour un salaire minimum d'environ 375 euros.

Selon le Bureau central d'information économique ${ }^{1}$ basé à Varsovie, au premier semestre de 2012, 1613 entreprises à capitaux étrangers étaient enregistrées en Pologne, dont 153 en Basse-Silésie (soit 9,40\%). La Basse-Silésie occupait ainsi la deuxième place derrière la région de la Mazovie (671 sociétés, soit $42 \%$ des entreprises à capitaux étrangers), devant la Silésie qui accueillait 147 entreprises, soit 9,11\%.

Depuis 2004, on observe une progression constante des investissements français en Pologne en valeur cumulée, ce qui place la France parmi les trois premiers investisseurs étrangers en Pologne (la position exacte diffère selon la source et le mode de définition de l'origine des capitaux). Présentes dans quasiment tous les secteurs d'activités, les plus grosses entreprises françaises sont cependant bien représentées dans la grande distribution (Auchan, Intermarché, Carrefour, Leclerc...), le secteur énergétique (Veolia, EDF, GDF Suez, Alstom Power), l'industrie et la construction (Lafarge, Saint-Gobain, Eurovia, Vinci, Egis, Systra, Rhodia), les services (Lagardère, France Télécom, Vivendi, Vinci, Bouygues, Accor, Canal+), le secteur bancaire (Crédit Agricole, Société Générale, BNP Paribas). Parmi les 25 plus gros investisseurs en Pologne, 8 sont d'ailleurs des entreprises françaises (Bertheau-Sobczyk 2015).

Située dans le sud-ouest de la Pologne, la Basse-Silésie présente en outre l'avantage de la proximité avec les pays de l'Ouest et avec ses voisins immédiats que sont la République tchèque et l'Allemagne. La région a bénéficié depuis 2005, comme l'ensemble de la Pologne, du développement particulièrement dynamique du secteur des services partagés (BPO/SSC) qui emploie déjà en 2015 plus de 150000 personnes à travers tout le pays. Wrocław est le troisième bassin d'emploi en importance dans ce secteur, derrière Varsovie et Cracovie avec 23000 employés. Dans ce secteur, la connaissance des langues étrangères est particulièrement recherchée. Bien qu'on ne dénombre que 42 entreprises françaises dans ce secteur sur les 649 recensées en 2015, nombre de sociétés polonaises et à capitaux étrangers ont également besoin de profils francophones.

\section{2. Études de philologie française à l'Université de Wrocław}

La première entité d'enseignement supérieur en Pologne consacrée à la recherche et à l'enseignement de langue et de civilisation françaises a été la Chaire de philologie romane (Katedra Filologii Romańskiej) fondée à Cracovie en 1892 à l'Université Jagellonne (Uniwersytet Jagielloński). À Wrocław, le premier département d’études romanes a été fondé en 1874 à l'Université allemande de Breslau (le nom allemand de la ville de Wrocław). Ce département a fonctionné jusqu'à la Seconde Guerre mondiale, époque à laquelle il a été fermé. Juste après la guerre, c'est l'Université de Lviv (Ukraine), déplacée de l'Est vers l'Ouest, qui occupa les locaux de l'ancienne université allemande. Durant 7 ans (de 1945 à 1952) des professeurs de l'université de Lviv ont assuré le fonctionnement du département de philologie romane: certains d'entre eux, qui venaient à Wrocław de temps en temps juste pour donner des cours, s'y installèrent. Ce département a été fermé en 1953 et après 7 ans de silence (inexis- 
tence d'un département d'études romanes à Wrocław), l'actuel Institut d'études romanes a été créé en 1960 et intégré à la structure de l'Université de Wrocław polonaise (voir Sawicki 1995: 222-225).

Pendant les trois années universitaires (2011-2012, 2012-2013, 2013-2014), desquelles provient l'échantillon analysé, il existait à l'IÉR deux types de diplômes de premier cycle (licence): les études françaises et les études hispaniques (trois ans) et de deuxième cycle (maîtrise, deux ans), en régime «de jour» (modalité gratuite, en semaine, destinée aux personnes qui ne travaillent pas) et en régime «week-end» (modalité payante, les week-ends, destinée aux personnes ayant un emploi). Les études de premier cycle ont été accessibles aux personnes avec un niveau de français débutant (filière $\mathrm{A}$ ) et avancé (filière $\mathrm{B}$ ). Au deuxième semestre de la deuxième année de licence, les deux filières se mélangeaient et un niveau commun B2+ a été créé. La formation en langue française termine à la fin de la troisième année au niveau $\mathrm{C}$.

Pendant l'époque analysée, les contenus spécifiques, propres à la formation en traduction français-polonais étaient insérés au cursus général de la formation philologique sous forme d'un module complémentaire nommé «module de traduction» (blok tłumaczeniowy)2.

Tous les étudiants en études françaises ou hispaniques apprenaient obligatoirement une deuxième langue romane (l'espagnol, le français, l'italien, le portugais ou le roumain) en plus de leur langue romane principale (le français ou l'espagnol, respectivement). Toutes les autres langues étrangères (y compris l'anglais et l'allemand) pouvaient être apprises comme matière supplémentaire, dans le cadre de crédits ECTS libres proposés aux étudiants.

Au deuxième semestre de la troisième année de licence, des stages professionnels obligatoires ( 2 crédits ECTS, 120 heures) étaient prévus dans le curriculum (à partir de l'année universitaire 2014-2015, ces stages ont été déplacés à la maîtrise). Suivant le décret no 56/2010 du recteur de l'Université de Wrocław du 29 juillet 2010, les stages pouvaient être réalisés soit sur la base d'une convention régulière signée entre le doyen et l'entité externe, soit en modalité de reconnaissance d'une activité professionnelle ou "préprofessionnelle» d'un étudiant, dûment documentée, d'une durée minimum de 120 heures et dont les contenus correspondaient aux compétences enseignées.

Jusqu'à présent (2015), pour les étudiants déjà inscrits à une université publique, il est possible en Pologne de poursuivre gratuitement ses études en vue de l'obtention d'un deuxième diplôme dans une université publique, mais ce règlement pourrait se voir modifié dans un proche avenir.

Dans le Tableau 1, nous présentons les détails de l'échantillon étudié ${ }^{3}$ et des données empiriques collectées. 
TABLEAU 1

Échantillon étudié et données empiriques collectées (philologie française, études de jour)

\begin{tabular}{|c|c|c|c|c|c|}
\hline $\begin{array}{l}\text { Aspect } \\
\text { analysé }\end{array}$ & $\begin{array}{l}\text { Année } \\
\text { universitaire } \\
\text { (semestre) de } \\
\text { collecte de } \\
\text { données }\end{array}$ & $\begin{array}{l}\text { Année } \\
\text { d'études des } \\
\text { étudiants, } \\
\text { filière }\end{array}$ & $\begin{array}{l}\text { Nombre } \\
\text { d'étudiants } \\
\text { dans } \\
\text { l'échantillon }\end{array}$ & $\begin{array}{l}\text { Nombre total } \\
\text { d'étudiants } \\
\text { inscrits dans } \\
\text { l'année } \\
\text { universitaire } \\
\text { (et filière) }\end{array}$ & $\begin{array}{l}\text { Technique de } \\
\text { collecte de } \\
\text { données et } \\
\text { objet étudié }\end{array}$ \\
\hline \multirow[t]{2}{*}{$\begin{array}{l}\text { Versant } \\
\text { «étudiants" }\end{array}$} & $\begin{array}{l}\text { 2011-2012 } \\
\text { (premier } \\
\text { semestre) }\end{array}$ & $\begin{array}{l}1^{\text {re }} \text { année de } \\
\text { licence, } \\
\text { filière } B\end{array}$ & 12 & $\begin{array}{l}73 \text { (dont } \\
\text { filière B 40) }\end{array}$ & $\begin{array}{l}\text { Enquête sur la } \\
\text { motivation } \\
\text { pour les } \\
\text { études et la } \\
\text { profession }\end{array}$ \\
\hline & $\begin{array}{l}\text { 2012-2013 } \\
\text { (premier } \\
\text { semestre) }\end{array}$ & $\begin{array}{l}1^{\text {re }} \text { année de } \\
\text { licence, } \\
\text { filière A }\end{array}$ & 48 & $\begin{array}{l}79 \text { (dont } \\
\text { filière A 47) }\end{array}$ & $\begin{array}{l}\text { Enquête sur le } \\
1^{\text {er }} \text { diplôme } \\
\text { (autre que la } \\
\text { philologie } \\
\text { française) }\end{array}$ \\
\hline \multirow[t]{3}{*}{$\begin{array}{l}\text { Versant } \\
\text { «employeurs» }\end{array}$} & $\begin{array}{l}2012-2013 \\
\text { (deuxième } \\
\text { semestre) }\end{array}$ & $\begin{array}{l}3^{\mathrm{e}} \text { année de } \\
\text { licence, filières } \\
\text { A et } \mathrm{B} \\
\text { mélangées }\end{array}$ & 28 & 36 & \multirow{2}{*}{$\begin{array}{l}\text { Analyse des } \\
\text { documents } \\
\text { des stages } \\
\text { professionnels } \\
\text { (non } \\
\text { pédagogiques) }\end{array}$} \\
\hline & $\begin{array}{l}\text { 2013-2014 } \\
\text { (deuxième } \\
\text { semestre) }\end{array}$ & $\begin{array}{l}3^{\mathrm{e}} \text { année de } \\
\text { licence, filières } \\
\text { A et } \mathrm{B} \\
\text { mélangées }\end{array}$ & 42 & 49 & \\
\hline & $\begin{array}{l}2012-2013 \\
2013-2014\end{array}$ & \multicolumn{3}{|c|}{$\begin{array}{l}60 \text { offres d'emploi au total provenant des } \\
47 \text { entités dirigées à tous les étudiants de l'IÉR } \\
\text { (licence + maîtrise) : } 50 \text { offres rédigées en } \\
\text { polonais }+10 \text { offres rédigées en anglais; } 42 \\
\text { entités recherchant du personnel francophone + } \\
5 \text { entités recherchant du personnel parlant } \\
\text { l'espagnol, l'italien ou le portugais }\end{array}$} & $\begin{array}{l}\text { Analyse des } \\
\text { documents } \\
\text { (offres } \\
\text { d'emploi) }\end{array}$ \\
\hline
\end{tabular}

\subsection{Correspondance entre la formation et l'emploi}

Bien qu'on puisse avoir l'impression que la formation en sciences humaines n'est pas actuellement une formation préférentielle aux yeux des autorités éducatives polonaises ${ }^{4}$ et européennes (vu la configuration des domaines pour le financement des projets de recherche Horizon 2020) ${ }^{5}$, l'insertion professionnelle des ressortissants des institutions d'enseignement supérieur dans les domaines langagiers, et plus spécifiquement dans le domaine de la traduction, occupe une place de plus en plus importante dans les actions universitaires et dans la recherche académique. Il suffit de citer la mise en place de nombreux outils servant à suivre le cheminement des diplômés (dont les enquêtes comme celles de l'Universitat Autònoma de Barcelona; voir Kuznik 2014a: 306); l'activité des groupes de recherche (GRET - Grup de Recerca en Educació i Treball, Universitat Autònoma de Barcelona); l'émergence des études dédiées à l'analyse de la recherche autour de l'insertion professionnelle des traducteurs (Torres 2010) et autour de la formation préparant à une telle insertion (Torres 2012; Marco Borillo 2016); la publication des études et réflexions qui soulignent l'importance des stages professionnels pour de futurs traducteurs (Astley 2013/2014; Kwiatek et Zabrocka 2014; Astley et Torres-Hostench sous presse; Kuznik 2014; Kuznik et Bestué 2016; Marco Borillo 2016); l'organisation de très nombreux colloques, récemment, au printemps 2015, ne serait-ce qu'en Pologne, consacrés à la 
problématique de la formation des spécialistes en langues pour le marché du travail (par exemple, le colloque «Od edukacji szkolnej do zatrudnienia. Kształcenie w języku francuskim», le 31 mars 2015, Uniwersytet Śląski, Katowice).

Évidemment, à part les voix en faveur de la formation supérieure (à long terme) soumise aux besoins (immédiats) du marché du travail, des voix plus critiques s'élèvent, notamment celles qui remettent en question l'indépendance (toujours relative) de l'enseignement supérieur (Dybiec-Gajer 2013; voir Kuznik 2014a: 304) et celles qui estiment que l'image de la situation sur le marché du travail n'est jamais claire ni univoque pour les services de traduction, ceci principalement à cause de la difficulté d'accéder aux données fiables sur le nombre d'employés dans le secteur de service en général, difficulté d'estimation quantitative des employés dans les services individualisés, difficulté d'accès et d'interprétation quantitative dans le cas des services sous-traités, des frontières fluides de la définition et donc de la délimitation des services langagiers (voir Kuznik 2014b : 2-3, 9-10).

Toutes les questions abordées ici relèvent d'une problématique beaucoup plus large et extrêmement importante qui peut se définir d'une manière très générale comme les politiques linguistiques (voir Gostkowska 2013: 9, 173-188). Cette problématique de fond ne peut pas être cependant exposée dans le cadre de ce travail plus limité. Il faut tout de même tenir compte du fait que cette correspondance entre la formation et les emplois à missions communicatives et langagières s'opère, dans notre cas, sur un territoire où le polonais, de famille slave occidentale, est la langue officielle d'État et la langue maternelle de la majorité de la population dont le statut européen et mondial est pourtant très différent de celui de la langue française, et encore plus de celui de la langue anglaise, l'anglais étant une langue hypercentrale, le français une langue supercentrale et le polonais une langue centrale, d'après la théorie sociolinguistique de Calvet (voir 1999: 76-79, cité aussi dans Gostkowska 2013: 9, 173-188).

\section{Versant " étudiants»}

Dans le versant "étudiants», nous avons mené deux enquêtes. La première a porté sur la motivation des étudiants de philologie française concernant leurs études et les métiers envisagés, et la deuxième a abordé le thème de la philologie française comme deuxième diplôme étudié.

\subsection{Méthodologie de collecte et d'analyse des données: deux enquêtes}

Le premier ensemble de données a été collecté pendant une enquête dans laquelle nous avons posé à nos étudiants (Tableau 1) trois questions ouvertes. Ils y répondaient par écrit de façon anonyme. Les questions étaient les suivantes:

1) Pourquoi avez-vous choisi la philologie française?

2) Comment vous imaginez-vous votre futur métier?

3) Quelles sont vos attentes par rapport à ces études? Comment imaginez-vous un programme d'études idéal?

Nous avons ensuite procédé à l'analyse du contenu des réponses en utilisant le logiciel EdEt Éditeur pour Ethnographes / Edytor Etnograficzny, version 1.99.920.1, 2.0 EN beta- $12^{6}$ développé par Iwona Kaliszewska de l'Institut d'ethnologie et d'anthropologie culturelle (Instytut Etnologii i Antropologii Kulturowej) et l'Institut 
d'informatique (Instytut Informatyki) de l'Université de Varsovie (Uniwersytet Warszawski). Nous avons réduit ce contenu aux principales catégories qualitatives. Les 12 étudiants y sont codifiés par les lettres d'A à L.

Ce logiciel permet d'organiser les différents types de fichiers (textes et images) et de procéder à une analyse qualitative de leurs contenus et à une analyse cohérente suivant les catégories d'analyse définies par le chercheur. Il a été conçu principalement pour l'analyse des études d'ethnologie et d'anthropologie (Kaliszewska 2009), avec une application prioritaire pour des entretiens à plusieurs interlocuteurs avec des caractéristiques très variées l'un de l'autre (plusieurs conversations en parallèle, groupes de discussion, focus group) mais il peut également être utilisé dans toutes les études où le chercheur doit travailler avec plusieurs répondants à la fois (personnes interrogées) ou plusieurs «voix» des interlocuteurs où des types de matériaux textuels sont combinés. EdEt permet, entre autres fonctions, d'assigner les catégories d'analyse (appelées «codes») aux fragments des textes choisis, de visualiser ces fragments avec trois couleurs différentes et de les exporter postérieurement dans un fichier .txt. Nous l'avons utilisé dans la recherche doctorale (voir Kuznik 2010/2012: 289) portant sur l'organisation du travail dans les entreprises de traduction. López-Sintas, Rojas, et al. (2015) et García-Álvarez, López-Sintas et al. (2015) se sont servis du même logiciel dans leurs études dans le domaine des sciences de la communication et des études interculturelles (voir aussi Kuznik, Verd et Olalla-Soler 2016).

Dans la deuxième enquête, orale cette fois, nous avons posé aux étudiants (Tableau 1) deux questions: s'ils étaient en train de suivre d'autres études supérieures en parallèle, et si oui dans quel établissement (UWr ou autre établissement d'enseignement supérieur), quels que soient le niveau d'études et les modalités (licence, maîtrise; études de jour, études de week-end). Le but de cette enquête était de savoir quels types d'étudiants (profils, affiliation, multiplicité de compétences) suivaient les études de philologie française comme deuxième formation ${ }^{7}$.

\subsection{Résultats et conclusions partielles du versant "étudiants"}

Les résultats détaillés du versant "étudiants» regroupés autour des aspects comme la multiplicité des langues, la configuration «en cascade» de la variété et du niveau des langues, le cas particulier de l'anglais, la flexibilité et la spécialisation des métiers envisagés, le cas de la traduction comme métier, et finalement les domaines des premières formations suivies en parallèle à la philologie française, sont présentés dans Kuznik (2015).

Comme conclusion du versant «étudiants», nous pouvons définir la motivation des étudiants en licence à poursuivre leurs études comme une motivation expressive car elle correspond à leurs rêves, leurs désirs, leurs passions, leurs intérêts personnels et leurs projections professionnelles. Cette motivation est expressive donc et ciblée aussi bien sur l'université (capacité et goût d'étudier) que sur la profession (vocation et contenus des métiers). Nous n'avons pas retrouvé de traces d'une motivation instrumentale qui aurait été ciblée soit sur l'université (l'importance ou la facilité d'obtention d'un diplôme) soit sur la profession (sécurité de l'emploi et d'une haute rémunération). Les étudiants veulent étudier la philologie française parce qu'ils adorent la langue, la culture et la littérature françaises et les langues en général. Nombreux sont ceux qui éprouvent et démontrent une vraie «soif des langues», une 
image qui correspond parfaitement à celle de la configuration des langues «en cascade», évoquée par nous dans Kuznik (voir 2015: 296-297). Nombreux sont aussi ceux qui se voient volontiers incorporés dans des entreprises comme traducteurs, dans leur avenir, l'image d'un traducteur corporatif étant la prépondérante dans notre échantillon, situation différente de celle identifiée par Cook (2012) dans son enquête menée parmi 117 étudiants de l'IÉR UWr (licence 78 étudiants, maîtrise avec spécialisation en traduction 14 étudiants et études post-diplôme en traduction des langues romanes 25 étudiants). Or, Cook constate que: «les étudiants actuels voudraient dans le futur devenir soit interprètes, soit traducteurs littéraires; certaines personnes, moins nombreuses, se montrent cependant intéressées par la traduction de textes spécialisés» (Cook 2012: 74).

Les étudiants conçoivent leur carrière professionnelle d'une manière cohérente, comme une continuation et une capitalisation des compétences déjà acquises, et une formulation successive des projets professionnels, ouverts, flexibles, avec des formations continues complémentaires de spécialisation. De cette façon, ils confirment la thèse exprimée par Planas (2011) suivant laquelle les horizons de projection ne sont pas forcément les mêmes pour les étudiants que pour les employeurs: les étudiants raisonnent à long terme, en temps de leur vie tandis que les employeurs raisonnent à court terme, en fonction de leurs besoins plutôt immédiats.

D’un point de vue quantitatif, nous estimons qu'environ la moitié des étudiants qui commencent la philologie française à l'IÉR dans les années universitaires étudiées sont des étudiants qui étudient également ailleurs pour obtenir leur premier diplôme. Ce phénomène signifie que ces étudiants enrichissent le milieu des études françaises par les informations, connaissances, lectures, méthodologies d'études et de travail, contacts sociaux et personnels de leurs domaines d'études d'origine, principalement d'autres langues étrangères, de la culture, de l'information et des médias, de l'économie et des relations internationales. Cet échange est à double sens : tous ces autres domaines restent imprégnés des mêmes éléments provenant des études romanes: la connaissance du français (et d'autres langues romanes), la littérature, l'histoire, la civilisation et la linguistique françaises.

Le fait de suivre simultanément deux formations universitaires rend les personnes polyvalentes, flexibles, les dote de compétences multiples, articulées autour de deux profils de compétences et en fait des personnes préparées pour un marché de travail changeant. Le potentiel des compétences est déjà présent, inscrit pendant l'étape de la formation supérieure. Il convient également de constater que les domaines généraux du premier diplôme ne sont pas très éloignés de la philologie française; ils constituent des zones d'intérêts communs et des carrefours des axes d'embauche tout à fait compatibles.

Le phénomène d'étudier en vue d'obtenir plus d'un diplôme à la fois apporte un argument en faveur de la constatation de Planas (2011) qui observe qu'à partir de la Seconde Guerre mondiale, chaque génération accède à un niveau d'éducation supérieur que la génération précédente. Cette circonstance crée à son tour un " effet offre», c'est-à-dire une situation qui favorise la hausse des exigences des employeurs car le niveau de formation augmente constamment dans les sociétés européennes issues et formées après la Seconde Guerre mondiale. 


\section{Versant "employeurs»}

Dans les sections ci-après nous présentons l'analyse des profils des stages et des premières expériences de travail des futurs philologues français aussi bien que les offres d'emploi s'adressant à tous les étudiants de l'IÉR UWr. Toutes ces sources de données nous renseignent sur les besoins actuels des employeurs dans la région de Wrocław cherchant à embaucher du personnel francophone.

\subsection{Méthodologie de collecte et d'analyse des données: analyse des documents}

Notre analyse des profils des stages, des premières expériences de travail et des offres d'emploi a consisté en une synthèse des informations contenues dans la documentation qui a accompagné la gestion des stages professionnels (Tableau 1). Les sources de données prises en compte ont été les suivantes:

- les documents qui établissent un lien formel entre les étudiants et/ou l'université et l'entité d'accueil: (1) la convention de stage et le programme du stage joint en annexe à la convention; (2) les différents types de contrats (contrat d'ouvrage ou de service, contrat de travail à durée déterminée ou indéterminée); (3) les accords de volontariat signés par les étudiants; (4) les accords internationaux entre universités;

- les attestations de stage délivrées par les entités d’accueil à la fin de la période de stage;

- les rapports de stage des étudiants rédigés par eux après cette expérience;

- les offres de travail (longs stages payants, travail saisonnier, emplois à court terme, postes fixes, etc.) reçues par le secrétariat de l'IÉR ou par le coordinateur des stages (auteur de cet article) et transmises aux étudiants moyennant le courrier électronique et l'affichage au tableau d'annonces.

\subsection{Résultats obtenus du versant «employeurs»}

\subsubsection{Profils des stages et des premières expériences de travail}

Nous analysons le profil des stages réalisés par nos étudiants et leurs premiers contrats de travail, d'ouvrage et de service reconnus par le coordinateur des stages à l'IÉR comme stage professionnel. Néanmoins, nous voulons attirer l'attention sur le fait que le nombre et le profil des stages professionnels ne sont pas forcément identiques au nombre et au profil des emplois réels existant sur le marché du travail à un moment donné (voir Kuznik 2014a: 311).

\subsubsection{Type de contrat}

Nous avons commencé notre analyse en regroupant les types de documents qui établissent un lien formel entre les étudiants et/ou l'université et l'entité d'accueil. Ceci nous a permis d'obtenir une liste des différents types de contrats, organisée de manière ascendante selon l'embauche de nos étudiants, des types de stages les moins susceptibles de donner lieu à leur embauche (faible embauche) aux différentes formes d'activités rémunérées (embauche élevée). Les données se présentent de la manière suivante:

- stages internes dans les différentes unités de l'UWr, notamment à l'IÉR et au Bureau des Relations Internationales, moyennant une convention de volontariat (faible embauche) - 16 étudiants, $23 \%$; 
- conventions de volontariat signées avec des associations (faible embauche) - 8 étudiants, $11 \%$;

- accords internationaux entre des universités (faible embauche) - 2 étudiants, $3 \%$;

- conventions régulières de stages (embauche élevée) - 22 étudiants, $31 \%$;

- contrats rémunérés d'ouvrage ou de service (embauche élevée) - 13 étudiants, $19 \%$;

- contrats de travail (à durée déterminée et indéterminée, embauche élevée) - 9 étudiants, $13 \%$.

Nous pouvons constater que plus d'un tiers de l'échantillon d'étudiants analysés $(32 \%=13 \%$ contrats de travail $+19 \%$ contrats d'ouvrage ou de service $)$ effectue déjà des activités d'une manière rémunérée. D’autres étudiants, représentant également légèrement plus d'un tiers (31\%, conventions régulières de stages), réalisent des stages avec un fort potentiel d'embauche. En général, à la fin de leurs études de licence, les étudiants en philologie française ont déjà une expérience du marché du travail et beaucoup d'entre eux y sont déjà pleinement intégrés.

\subsubsection{Pays et localité}

Les stages se sont déroulés principalement en Pologne (62 cas, $89 \%$ ), avec une intéressante incidence des stages réalisés en France (6 cas, 9\%). Nous observons aussi une présence d'autres pays situés sur d'autres continents mais cette participation doit être considérée comme plutôt anecdotique (Tunisie 1 cas, $1 \%$; Vietnam 1 cas, $1 \%$ ). Les stages réalisés sur le territoire français sont des types suivants: 3 contrats de travail, 2 contrats d'ouvrage ou de service et 1 accord international.

Si nous nous concentrons sur les localités situées sur le territoire polonais et les entités d'accueil à haut potentiel d'embauche des étudiants (Tableau 2), nous obtenons les résultats pour les 38 étudiants qui se répartissant selon les types de stages suivants:

- contrats de travail: 5 en Pologne (9 dans l'échantillon);

- contrats d'ouvrage ou de service: 11 en Pologne (13 dans l'échantillon);

- conventions de stage régulières: 22 en Pologne (22 dans l'échantillon).

TABLEAU 2

Localités des stages réalisés en Pologne, dans des entités d'accueil avec un haut potentiel d'embauche

\begin{tabular}{|c|c|c|}
\hline Localité & Région & Nombre d'étudiants \\
\hline Wrocław (approx. $71 \%$ ) & \multirow{4}{*}{$\begin{array}{c}\text { Basse-Silésie } \\
\text { (województwo dolnoślaskie) }\end{array}$} & 27 \\
\hline Dzierżoniów & & 1 \\
\hline Oława & & 1 \\
\hline Karpacz & & 1 \\
\hline Niemodlin & $\begin{array}{c}\text { Région d'Opole } \\
\text { (województwo opolskie) }\end{array}$ & 1 \\
\hline Zabrze & \multirow{3}{*}{$\begin{array}{c}\text { Haute-Silésie } \\
\text { (województwo śląskie) }\end{array}$} & 1 \\
\hline Knurów & & 1 \\
\hline Ożarowice & & 1 \\
\hline Kraków-Zabierzów & $\begin{array}{c}\text { Petite-Pologne } \\
\text { (województwo małopolskie) }\end{array}$ & 1 \\
\hline Rzeszów & $\begin{array}{c}\text { Basses-Carpates } \\
\text { (województwo podkarpackie) }\end{array}$ & 1 \\
\hline Warszawa & $\begin{array}{c}\text { Mazovie } \\
\text { (województwo mazowieckie) }\end{array}$ & 1 \\
\hline Olsztyn & $\begin{array}{c}\text { Varmie-Mazurie } \\
\text { (województwo warmińsko-mazurskie) }\end{array}$ & 1 \\
\hline Total & & 38 \\
\hline
\end{tabular}


D’après ces résultats, les entités d'accueil à haut potentiel d'embauche de nos étudiants se trouvent principalement dans le sud de la Pologne (36 étudiants sur 38), sont réparties entre la ville de Wrocław et sa région (30 étudiants, $71 \%$ environ) et aussi dans d'autres zones du sud de la Pologne (la région d'Opole, la Haute-Silésie, la Petite-Pologne et les Basses-Carpates). Sporadiquement, et cela grâce à leurs contacts personnels, les étudiants réalisent leur stage avec un potentiel intéressant d'embauche autour de la capitale de la Pologne et dans le nord de la région.

\subsubsection{Domaines de l'activité économique. Activités effectuées pendant les} stages dans les entités d'accueil en Pologne

Nous avons analysé la documentation relative aux 38 stages (dossiers de 38 étudiants) effectués dans les entités d'accueil en Pologne présentant un haut potentiel d'embauche (les conventions ordinaires de stage et les différents types de contrats: contrat d'ouvrage ou de service, contrat de travail à durée déterminée ou indéterminée, Tableau 3 dans l'Annexe 1). Nous avons exclu de cette analyse les formes de stages réalisés en Pologne et à l'étranger présentant de faibles possibilités d'embauche future.

\subsubsection{La variété organisationnelle des entités d'accueil}

Grâce aux données réunies dans l'Annexe de cet article (Tableau 3), nous pouvons constater une grande variété organisationnelle des profils des entités qui ont accueilli les 38 étudiants pour les stages. Nous y retrouvons aussi bien de grands groupes internationaux à capitaux étrangers (par exemple HCL Poland, Ernst \& Young Global Services Poland, Telecommunications Operations \& Service Centre / ACN) que des petites et moyennes entreprises polonaises (par exemple El Universo / Przystek Edu ou Laboratorium Analityczne Diagnoza). Il existe même des cas de PME créées par d'anciens étudiants en philologie française, des cas d'activité économique indépendante (El Sueño) et des représentants des professions libérales (Kancelaria Adwokacka). Parmi ces entités, nous comptons aussi quatre entités publiques (Dolnośląska Biblioteka Publiczna / Biblioteka Romańska, Alliance Française - Stowarzyszenie Wrocław, Przedszkole Nr 147 we Wrocławiu, Uniwersytet Warmińsko-Mazurski / Instytut Słowiańszczyzny Wschodniej) disposant d'un système d'embauche spécifique. Dans la majorité des cas, les entités accueillent un seul étudiant, sporadiquement deux, pendant une période de deux années universitaires.

\subsubsection{La multiplicité des langues de travail}

En ce qui concerne les langues de travail mentionnées dans la documentation des stages (Tableau 3 dans l'Annexe 1), la langue française est évidemment prépondérante, avec une forte présence de la langue anglaise (dans 13 cas) et la présence plus faible d'autres langues romanes (l'espagnol - 5 cas; l'italien - 1 cas) et une apparition sporadique de la langue allemande $(2 \mathrm{cas})$. Il n'est cependant pas rare que nos étudiants utilisent plus d'une langue étrangère (14 cas), voire jusqu'à un maximum de quatre langues étrangères (stage au Corner Hostel dans le domaine du tourisme). 


\subsubsection{La nature multidimensionnelle des domaines d'activité pendant les stages}

Nous avons identifié les domaines économiques suivants dans lesquels s’inscrivent les activités effectuées par nos 38 étudiants pendant les stages (Tableau 3 dans l'Annexe 1):

- culture - 2 étudiants;

- éducation - 7 étudiants;

- gestion comptable et financière - 3 étudiants;

- gestion des ressources humaines - 1 étudiant;

- gestion des ventes et marketing - 2 étudiants;

- publicité (arts visuels numériques) - 3 étudiants;

- télécommunications, internet, produits multimédias - 4 étudiants;

- transport aérien - 4 étudiants;

- hôtellerie (tourisme) - 2 étudiants;

- restauration (culture, tourisme) - 2 étudiants;

- traduction - 7 étudiants;

- support juridique (documentation) - 1 étudiant.

Il faut néanmoins souligner que cette classification n'est qu'une tentative assez arbitraire; en fait, cette réalité est multidimensionnelle. À part un premier domaine d'activité dominant, on peut toujours discerner un deuxième ou un troisième domaine potentiel, latent. En plus, dans la documentation analysée d'une manière inductive, nous retrouvons aussi bien des domaines relevant d'un champ thématique concret (télécommunications, internet, produits multimédias, transport aérien, hôtellerie, restauration, etc.) que des domaines en principe «vides» de contenus et pouvant desservir n'importe quel autre domaine particulier (éducation, gestion, publicité, traduction, etc.).

La culture en particulier semble omniprésente: elle marque de sa présence toutes ces activités d'une façon ou d'une autre. L'étudiant qui a réalisé son stage au Bistrot Parisien a souligné que son activité de serveur avait été enrichie par une activité de consultant culturel (comparaison des habitudes gastronomiques des Français et des Polonais, confection des menus, etc.). De la même manière, l'étudiant en stage à l'école maternelle $\mathrm{n}^{\circ} 147$ a transmis pendant ses activités didactiques des contenus centrés sur la culture française (éléments de culture française contenus dans des chansons pour enfants).

En touchant à toutes les tâches et fonctions liées à la gestion de l'entreprise (produits financiers, comptabilité, ressources humaines, marketing, etc.), nos étudiants acquièrent des connaissances et compétences entrepreneuriales, absentes en principe du programme d'études actuel.

Finalement, le domaine du transport aérien nous semble d'un intérêt particulier, car 4 étudiants ont été embauchés pendant les stages, ce qui n’a pas été le cas des transports routier, ferroviaire, maritime ou fluvial, totalement absents de cette base de données.

\subsubsection{Le cas de la traduction}

La traduction constitue un domaine très important parmi les activités de stage (Tableau 3, en annexe). Elle semble être l'activité de base dans 7 cas, et dans 7 autres cas, elle est mentionnée comme composante d'un autre domaine (culture, gestion 
des ventes et marketing, publicité, transport aérien, restauration). L'intérêt pour la traduction assermentée est évident: même un stage dans un cabinet d'avocat peut être envisagé comme une préparation professionnelle pour ce métier.

Dans les 7 premiers cas (domaine: traduction), les traductions sont accompagnées d'activités de gestion de bureau de traduction, de gestion des projets de traduction et de service au client. Toutes ces activités se complètent pour créer un faisceau d'activités difficilement séparables.

\subsubsection{Offres d'emploi}

Dans les sections suivantes, nous exposons quelques grandes lignes d'un aperçu général sur le contenu des offres d'emploi ${ }^{8}$ adressées aux étudiants (Tableau 1) et nous montrons si et dans quelle mesure le travail pour les traducteurs y est présent.

\subsubsection{Dédoublement d'affichages provenant des employeurs directs} et des entreprises d'embauche

Une première remarque que nous pouvons faire, après une analyse générale du contenu des offres d'emploi, concerne le doublonnage d'offres provenant des employeurs directs et des entreprises d'embauche comme Adecco Poland, Becton \& Dickinson Recruitment, Grafton Recruitment, CPL Jobs, Goldman Recruitment, IES Consulting, Manpower, Otto Praca, Power Media, StaffPoland, etc. Il faut être conscient que sur ces 60 offres d'emploi reçues, beaucoup d'entre elles sont des doublons. Le concours des intermédiaires sur le marché de travail brouille considérablement l'image de la situation dans ce secteur, outre le fait qu'il contribue évidemment, d'une manière positive, à l'intensification de la recherche de l'emploi.

\subsubsection{Langues des offres d'emploi et langues des emplois:}

le français et l'anglais

Sur les 60 offres, une dizaine d'offres est rédigée uniquement en anglais, même si ce sont des personnes qui parlent le français qui sont recherchées. L'analyse des tendances générales qui suit se réfère seulement aux 50 offres d'emploi rédigées en polonais.

La combinaison de langues la plus recherchée est le français et l'anglais (niveau de communication). Le français est rarement demandé seul. Sur les 47 entités, seules 5 ne requièrent pas le français mais cherchent d'autres langues romanes (espagnol, italien et portugais). Les 42 entités restantes veulent embaucher des personnes qui parlent français.

\subsubsection{Tendances générales par domaines d'activité}

Dans l'analyse sommaire qui suit, nous avons identifié les tendances générales par domaine d'activité économique (il faut néanmoins tenir compte que les entités des stages et les entités qui offrent un travail à nos étudiants peuvent être les mêmes).

- Nous avons pu constater la présence des PME locales de la région de Wrocław, comme Zapach Lawendy ou Gambit Lubawka, qui, pour la réalisation de leurs stratégies d'internationalisation, ont besoin d'un personnel multilingue. Parfois, derrière une annonce affichant un visuel peu engageant (qui suggérerait l'existence d'une PME aux moyens économiques très restreints) se cache un travail très enrichissant et une société locale très dynamique; 
- L'externalisation des processus d'affaires (la comptabilité, principalement) continue à être un domaine d'activité très recherché, en particulier par des grands groupes internationaux, mais toutes les activités liées à la gestion des ventes et du marketing semblent très présentes, surtout dans le cas des PME locales à vocation internationale;

- La présence très visible de la logistique et du transport aérien se confirme (Sky Taxi, Enter Air), enrichie par la présence du transport routier (Kuehne + Nagel);

- La gestion des ressources humaines au sein des entreprises et en particulier la sélection du personnel parlant des langues étrangères constitue un domaine d'activité très sollicité;

- Le tourisme affirme sa présence très nette dans les offres d'emploi, cette fois-ci sous la forme du tourisme d'accueil et, une nouveauté, de l'animation du temps libre (Stageman);

- Dans les offres d'emploi collectées, l'enseignement des langues étrangères et des activités autour de l'éducation en général se veut dans des environnements informatisés et en ligne;

- Finalement, une dernière remarque dans cette analyse sommaire: les activités professionnelles relatives à la culture passent plutôt par des canaux institutionnels (Academia Europaea, Alliance Française).

\subsubsection{Le cas de la traduction}

Parmi les 60 offres d'emploi, nous trouvons très peu d'offres pour les traducteurs, la traduction et l'interprétariat étant plutôt insérés dans les activités propres du service clientèle et de la gestion générale de l'entreprise. À ce propos, nous citons deux cas particuliers d'offres d'emploi dans le domaine de la traduction et de l'interprétariat: l'offre de la maison Translativc qui recherche un chef de projet dans une offre rédigée complètement en anglais et l'association Kraina Łęgów Odrzańskich - Stowarzyszenie Lokalne Grupa Działania, qui recherche un traducteur-interprète pour un déplacement en France à l'été 2014. Comme dans le cas de premières expériences de travail de nos étudiants en traduction, parmi les offres de travail pour les traducteurs et les interprètes collectées, le caractère hybride, soit les contenus très hétérogènes de ces postes, saute immédiatement aux yeux.

\subsection{Conclusions partielles du versant «employeurs»}

\subsubsection{Insertion professionnelle à la fin des études de premier cycle}

L'analyse réalisée nous permet de constater que les étudiants qui ont fait des études de français sont présents sur le marché du travail local avant la fin de leurs études de premier cycle: plus d'un tiers de l'échantillon d'étudiants analysé (32\%) effectue déjà des activités rémunérées (contrats de travail et contrats d'ouvrage ou de service) et également plus d'un tiers (31\%) réalise des stages à fort potentiel d'embauche.

Cette constatation confirme les résultats d'une enquête effectuée en 2004/2005 par l'Association académique des romanistes polonais «Plejada », avec le soutien du ministère des Affaires étrangères français, de l'ambassade de France en Pologne et du Centre de civilisation française parmi les diplômés des philologies romanes (promotions des années 2000-2003, échantillon couvrant toute la Pologne; 554 réponses obtenues soit $37 \%$ des diplômés des années 2000-2003; voir Skibińska 2007: 90-92). Ladite enquête a été organisée par la présidence de cette Association non seulement du point de vue financier, mais aussi du point de vue organisationnel et de la conception de l'ensemble. 
Les résultats du projet mené par l'Association montrent en général une bonne insertion professionnelle des philologues français sur le territoire polonais au début du $\mathrm{XxI}^{\mathrm{e}}$ siècle: parmi d'autres résultats obtenus et exposés par le sociologue Tadeusz Sozański dans son rapport interne non publié, $13 \%$ des diplômés ont déjà été embauchés sous contrat ponctuel de prestation de services et 13,8\% des diplômés ont trouvé un travail avant la fin de leurs études de maîtrise (voir Skibińska 2007: 91).

Les conclusions formulées par Cook (voir 2012: 66) s'inscrivent dans la même ligne en ce qui concerne l'insertion professionnelle des étudiants de philologie française de l'IÉR UWr de niveau licence inscrits au module de traduction. Cook constate que $6 \%$ des étudiants interrogés (78 étudiants en licence, sur un total de 117 étudiants interrogés) travaillent déjà en tant que traducteurs.

Par ailleurs, l'efficacité des stages dans le processus d'insertion professionnelle des diplômés des études spécialisées en traduction, dans d'autres pays européens et à un niveau supérieur au premier cycle, a déjà été démontrée par Astley (2013/2014), Kwiatek et Zabrocka (voir 2014: 352), Astley et Torres-Hostench (sous presse) pour les études de maîtrise en traduction et en gestion de projets de traduction (le projet des stages professionnels dans des entreprises de traduction - the European Graduate Placement Scheme) et par Pym, González Núñez et al. (2014) pour les études de doctorat (huit différents projets de transfert des connaissances dans le domaine de la traduction écrite et audiovisuelle, réalisés en coopération entre l'université et les entreprises, soit les organisations inter- et non gouvernementales).

\subsubsection{Nature hybride des postes de traducteurs}

Aux résultats obtenus par Sozański et Skibińska (voir 2007: 90-94), on peut ajouter les données recueillies et les conclusions formulées à l'issue d'une enquête menée à l'IÉR UWr parmi les 82 titulaires de certificats (délivrés depuis 1994) de formation spécifique à la traduction français-polonais, intégrée dans le cursus philologique au niveau de la maîtrise (4 réponses obtenues, soit 67,1\%). Skibińska affirme que:

La demande de traduction du français n'est cependant pas suffisante pour constituer la source principale des revenus de tous ceux qui le souhaiteraient [...]. Remarquons que parmi les personnes qui utilisent régulièrement leurs compétences de traducteur, 11 travaillent dans des entreprises où il s'agit de faire aussi autre chose que traduire (ou plutôt, la traduction, est une tâche annexe se superposant à des tâches plus fondamentales). (Skibińska 2007: 94)

Cette nature hybride des postes de travail des traducteurs, étudiée et décrite à plusieurs reprises (Kuznik et Verd 2010; Kuznik 2010/2012, 2011, 2016) et détectée également dans la présente analyse, avait été déjà identifiée par Bowker (2004), dans le contexte canadien du début du $\mathrm{XXI}^{\mathrm{e}}$ siècle, pour toutes les langues incluses dans son corpus d'offres d'emploi.

\subsubsection{Intérêt et initiative des groupes internationaux et des PME}

Le fait d'avoir analysé 60 offres d'emploi reçues ${ }^{10}$ par l'IÉR montre aussi quelles entités de la région de Wrocław sont à présent les plus dynamiques. Notre échantillon nous fournit une information fiable sur l'intérêt et l'initiative des organismes locaux dont les besoins en embauche de personnel francophone ne sont pas couverts. Or, ce sont majoritairement des grands groupes internationaux délocalisés (offshored), avec 
leurs centres de services partagés (shared service centres) situés dans la région de Wrocław, pour lesquels nos étudiants effectuent des activités d'externalisation des processus d'affaires (business process outsourcing, BPO) et desservent de la Pologne leurs clients du marché français principalement. Comme deuxième groupe d'entités dynamiques, nous pouvons citer des PME locales qui s'initient à leurs processus d'internationalisation.

De manière surprenante, nous constatons l'absence dans notre base de données (années 2011-2014) des entités suivantes à capital français, situées dans la région de Wrocław et de la Basse-Silésie, associées à la Chambre de Commerce et d'Industrie Française en Pologne (par ordre alphabétique): Anis Opakowania, Avanta Audit, Carefleet, Credit Agricole Bank Polska, Custo Pol, Europejski Fundusz Leasingowy, Forest Logistyka, Hologram Industries Polska, Kancelaria Prawna Schampera, Dubis, Zając i Wspólnicy, Profes, Standis Polska, Systra Oddział w Polsce et Work Service ${ }^{11}$. Il est probable que toutes ces entités, présentes sur le territoire polonais depuis longtemps, aient déjà établi leurs propres protocoles d'embauche interne et jouissent d'un réseau de contacts beaucoup plus solide et étendu que d'autres entreprises (groupes internationaux à capital autre que français et PME polonaises).

\section{Conclusions}

Dans cette analyse inductive, nous avons porté notre intérêt de recherche sur les caractéristiques particulières de l'insertion professionnelle des étudiants et des diplômés des études de philologie française, des traducteurs inclus, au niveau de la licence, inscrits à l'Institut d'études romanes de l'Université de Wrocław, dans la région sudouest de la Pologne, entre 2011 et 2014. Les conclusions de notre étude peuvent se résumer de la façon suivante:

\subsection{Considérations méthodologiques}

I. La pertinence de la généralisation des résultats provenant des études de cas a toujours suscité une polémique parmi les chercheurs et les méthodologues de la recherche. Suivant Creswell (2003), dans ce type de stratégie méthodologique, « [...] the researcher explores in depth a program, an event, an activity, a process, or one or more individuals. The case(s) are bounded by time and activity, and researchers collect detailed information using a variety of data collection procedures over a sustained period of time» (Creswell 2003: 15). Yin (1994), dans sa définition des études de cas, met en avant l'influence de l'information contextuelle dans laquelle chaque cas est forcément submergé: "A case study is an empirical inquiry that investigates a contemporary phenomenon within its real-life context, especially when the boundaries between phenomenon and context are not clearly evident» (Yin 1994: 13). L'auteur ajoute ensuite que «[...] phenomenon and context are not always distinguishable in real-life situations» (Yin 1994: 13). Pour argumenter la pertinence de la généralisation des résultats obtenus dans des études de cas, Yin oppose la généralisation analytique («analytic generalization») à la généralisation statistique («statistical generalization») dans la formulation suivante: «[...] case studies [...] are generalizable to theoretical propositions and not to populations or universe. [...] [The case study] does not represent a 'sample' [...], and your goal will be to expand and 
generalize theories (analytic generalization) and not to enumerate frequencies (statistical generalization)» (Yin 1994: 10).

II. Dans notre description de la situation qui a réellement eu lieu dans un contexte régional d'un pays centre-européen et pendant la période étudiée, nous avons essayé d'assurer la complémentarité des sources et des formats de données aussi bien que des techniques de collecte et d'analyse des données pour que les résultats obtenus soient les plus complets possible. D’une manière spéciale, le fait de réunir dans la même étude la vision des étudiants (versant "étudiants») et celle des employeurs (versant «employeurs») devrait contribuer à ce caractère complet et équilibré de nos résultats. Suivant Rojo (voir 2013: 43-44), la plénitude et la profondeur des études de cas, obtenues grâce à l'intérêt porté aux caractéristiques particulières de l'objet de l'étude, constituent l'un des principaux avantages de ce type de stratégie méthodologique.

III. Flyvbjerg (2004), dans sa défense des études de cas, associe ce type de démarche méthodologique à des notions de contexte, profondeur, complexité, ambigüité, richesse, expérience et apprentissage. Il souligne le besoin de produire des études de cas dans chaque discipline scientifique, et surtout dans les sciences sociales, car une discipline, qui s'intéresse à l'activité de l'homme, sans un nombre considérable de descriptions profondes des phénomènes existant dans la réalité (des «exemplaires de la réalité») serait une discipline morte. En plus, en s'appuyant sur d'autres auteurs (Bourdieu, Dreyfus, Christensen, Hansen, Cragg, Campbell, Eysenck, Ragin et Becker), Flyvbjerg affirme que les études de cas sont cruciales dans la construction du savoir humain expert car les connaissances d'une personne débutant dans une activité émanent de l'apprentissage des normes et des règles tandis que celles d'un expert sont dues à l'assimilation des milliers des cas particuliers, contextualisés et transformés en un savoir tacite spécialisé (voir Flyvbjerg 2004: 36-39).

\subsection{Considérations thématiques}

I. Les langues et les activités envisagées par les étudiants et par les employeurs se structurent "en cascade», avec une superposition évidente des niveaux de langues étrangères et des tâches à accomplir, une cascade multilingue et multitâche.

II. La traduction dans le domaine francophone présente une image ambiguë: elle est très désirée par les étudiants comme métier, présente au cours des stages et des premiers jobs à part entière ou bien insérée à d'autres missions, mais elle est quasiment absente dans les offres d'emploi reçues.

III. Les domaines de l'activité économique forment plusieurs axes d'embauche intéressants, grâce aux zones d'intersection des données empiriques provenant, d'un côté, du versant "étudiants", et, de l'autre côte, du versant "employeurs" réels et potentiels (Figure 1). Cette image nous paraît tout à fait cohérente aussi bien dans une lecture horizontale, par source de données, que dans une lecture verticale, par axes d'embauche.

IV. À l'issue de la licence, les étudiants sont intégrés dans le marché du travail local et régional. Une certaine évolution de leurs projets professionnels est cependant observable. Les étudiants commencent en première année à avoir des idées sur leur avenir professionnel, des idées bien ancrées dans la réalité qui les entoure. Très nombreux sont ceux qui ajoutent les compétences acquises pendant leurs études en phi- 
FIGURE 1

Tableau synoptique des domaines d'activité économique des philologues français (voir aussi Kuznik 2015, Tableau 5)

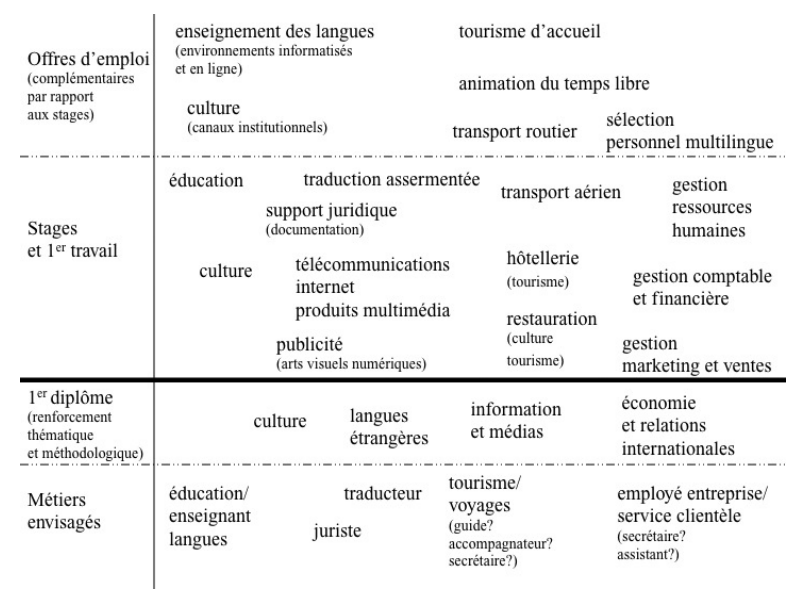

lologie française aux compétences des premiers diplômes déjà obtenus. Les stages en troisième année montrent nettement que le domaine économique d'embauche projeté au début des études se concrétise sous l'effet du contact direct avec le tissu économique, entrepreneurial externe à l'université, s'enrichit et se diversifie. L'irruption des entreprises et des institutions dans le quotidien universitaire provoque une explosion et une dispersion typologique et géographique de ces domaines. De cette manière, la configuration des domaines économiques visible dans la Figure 1 prend la forme d'entonnoirs (ou panaches) dont les premières projections, idées et motivations forment la base.

V. Les jeunes croient en leur avenir professionnel et en leur formation en langues, et les entités dynamiques et actives de la région trouvent le moyen de rencontrer ces jeunes. Dans bien des cas, l'offre d'emploi répond à la demande. Une étude approfondie de l'insertion réelle (et non seulement de l'embauche) à une échelle plus large, qui prendrait en compte la mobilité géographique des jeunes après leurs études et l'impact du facteur «genre» (homme/femme), vu qu'il s'agit d'études et de métiers fortement féminisés, serait souhaitable. Une étude plus quantitative, systématique et structurée portant sur les activités effectuées par l'étudiant (traduction, rédaction, service à la clientèle, formation) d'une part et d'autre part sur le domaine de l'activité principale de la société (transport, télécommunications, gestion) constituerait également une intéressante perspective de recherche.

VI. De plus, il nous reste à analyser en détail les offres d'emploi pour savoir à quel niveau se situent les connaissances approfondies et avancées relativement aux besoins des employeurs. Nous avons l'impression que les employeurs demandent des jeunes une maturité personnelle et psychologique tout en offrant des activités professionnelles qu'il est possible d'effectuer juste après la licence. Alors, qui a besoin de nos spécialistes, et où? Notre future recherche pourrait apporter des éléments de réponse à cette question. 


\section{REMERCIEMENTS}

Les auteures de cet article tiennent à remercier Maryline Bertheau-Sobczyk, présidente de Valians International Sp. zo.o. de Cracovie pour nous avoir transmis les données de contextualisation économique; Iwona Kaliszewska de l'Institut d'ethnologie et d'anthropologie culturelle de l'Université de Varsovie pour son autorisation d'utiliser le logiciel EdEt; et Jérôme Drobinsky du Credit Suisse Centre d'excellence de Wrocław pour une première lecture critique de ce texte.

\section{NOTES}

1. Le Bureau central d'information économique (Centralny Ośrodek Informacji Gospodarczej): page consultée le 18 août 2013, www.coig.com.pl/

2. Pour l'incorporation de la formation propre à la traduction dans le cursus générale philologique à l'IÉR UWr, voir Skibińska et Kaufman 2000, Skibińska 2011; 2007. La formation à la traduction professionnelle se concentre principalement au niveau des études de maîtrise.

3. Pour préciser la portée de l'échantillon, nous donnons ci-après les nombres moyens totaux d'étudiants inscrits, résumant les trois années universitaires 2011-2012, 2012-2013 et 2013-2014 (état au 30 novembre de 2011, 2012 et 2013) : nombre moyen d'étudiants inscrits à l'Université de Wrocław : 28784 (études de jour 19401 + études de week-end 9 383); nombre moyen d'étudiants inscrits à la Faculté des lettres: 6881 (études de jour 4665 + études de week-end 2.216); nombre moyen d'étudiants inscrits à la philologie française (licence et maîtrise) : 278 (études de jour 253 + études de week-end 25) (UWr 2011, 2012, 2013).

4. Dans un des concours publics, lancé par le Centre national de recherche et développement polonais (Narodowe Centrum Badań i Rozwoju) le 6 août 2012 pour accorder des subventions supplémentaires aux champs de formation préférentiels (stratégiques, cruciaux), les domaines des sciences humaines sont cruellement absents (sauf, peut-être le point 4 . Industries de la culture). Par contre, on y retrouve les 11 domaines comme suit (par ordre alphabétique en polonais): 1. architecture, urbanisme, arrangement des intérieurs; 2 . solutions technologiques dans la conception des processus industriels : automatisation, robotique, biotechnologie, bio-informatique, génie biomédical, génie chimique et des processus, mécatronique, nanotechnologies; 3 . bâtiment, génie civil des ponts et chaussées, geo-ingénierie; 4 . industries de la culture, éducation artistique en arts plastiques, en réalisation des images télévisées, des films et des photographies; 5 . technique et informatique; 6 . électronique et télécommunications; 7 . énergie et recherche des sources d'énergie; 8 . génie et protection de l'environnement; 9. logistique; 10. gestion et technologies de la production dans l'industrie, incluant l'industrie du bâtiment, l'industrie chimique, l'industrie de la transformation (des aliments) et de l'agriculture; 11 . médecine et assistance aux personnes âgées, infirmerie, physiothérapie, gériatrie, thérapie génétique.

5. Horizon 2020. The EU Framework Programme for Research and Innovation: page consultée le 15 mai 2016, https://ec.europa.eu/programmes/horizon2020/en/find-your-area

6. Le logiciel EdEt a été téléchargé sur le site web www.etnologia.uw.edu.pl/etno/dlaStudentow/edet. Trois versions linguistiques sont accessibles: polonaise, anglaise et espagnole.

7. Dans la première enquête aux étudiants de la première année, portant sur leur motivation d'étudier la philologie française et leur vision du futur métier, 3 étudiants ont mentionné les diplômes et les formations complémentaires à suivre après la philologie romane, et ce sont: marketing ou relations économiques internationales (étudiant E); tourisme (étudiant K); et culture européenne (étudiant L) (Kuznik 2015: 293-295, Tableau 1).

8. Pour l'évolution du modèle discursif de l'offre d'emploi en Pologne après 1989, ses réalisations polonaise (sur un corpus d'offres d'emploi parues dans la presse polonaise entre 1988 et 2001) et française (sur un corpus d'offres d'emploi parues dans la presse française entre 1993 et 2001), et leur étude contrastive, consulter Skibińska 2002a; 2002b. Pour l'utilisation de ces deux corpus en tant que textes parallèles dans une classe de traduction français-polonais, consulter Skibińska 2002c.

9. Pour plus de détails sur cette enquête, voir le site web de l'Association académique des romanistes polonais (Akademickie Towarzystwo Romanistów Polskich) «Plejada» : page consultée le 15 mai 2016, http://neo.amu.edu.pl/plejada/index.php/fr

10. Le coordinateur des stages qui est aussi la personne chargée de recevoir et de diffuser les offres d'emploi parmi les étudiants est déjà suffisamment occupé par les offres d'emploi «entrantes» pour ne pas chercher de contacts externes, à sa propre initiative et selon ses propres critères, ce qui est probablement dommage. 
11. Chambre de Commerce et d'Industrie Française (Francusko-polska Izba Gospodarcza): page consultée le 12 octobre 2013, www.ccifp.pl

\section{RÉFÉRENCES}

AstLey, Helen (2013/2014): Translating study into jobs. The Linguist. 52(6):20-21.

Astley, Helen et Torres-Hostench, Olga (sous presse): The European Graduate Placement Scheme: an integrated approach to preparing Masters in Translation Graduates for employment. The Interpreter and Translator Trainer, numéro spécial «Employability and the Translation Curriculum» sous la direction de Begoña Rodríguez de Céspedes, Jonathan Evans et Akiko Sakamoto (vol. 11, n 2, 2017).

Bertheau-Sobczyk, Maryline (2015): Znajomość języka francuskiego w relacjach pomiędzy firmami francuskimi i polskimi. / Biznesowe relacje pomiędzy Polską a Francją (PPT) (Od edukacji szkolnej do zatrudnienia. Kształcenie w języku francuskim, Uniwersytet Śląski, Katowice, 31 mars 2015). Communication orale avec diaporama.

Bowker, Lynne (2004): What Does It Take to Work in the Translation Profession in Canada in the 21st Century? Exploring a database of Job Advertisements. Meta: Translators' Journal. 49(4):960-972.

Calvet, Louis-Jean (1999): Pour une écologie des langues du monde. Paris: Plon.

Cook, Jadwiga (2012): Figure de rêve ou comment les étudiants se figurent le traducteur. Romanica Wratislaviensia. LIX:65-75.

Creswell, John W. (2003): Research Design: Qualitative, Quantitative, and Mixed Approaches. Thousand Oaks, CA: Sage.

Dy biec-Gajer, Joanna (2013) : Tłumaczenie jako świadczenie usług tłumaczeniowych? Między realizmem zawodowym a dyktatem rynku w zakresie kształcenia tłumaczy. Między Oryginałem a Przekładem. 19-20:171-189.

Flyvbjerg, Bent (2004): Cinco malentendidos acerca de la investigación mediante los estudios de casos. Reis. 106/04:33-62.

García-Álvarez, Ercilia, López-Sintas, Jordi et Samper-Martínez, Alexandra (2015): The Social Network Gamer's Experience of Play: A Netnography of Restaurant City on Facebook. Games and Culture. July 20, 2015: 21 p. Page consultée le 30 décembre 2015, <http:// gac.sagepub.com/content/early/2015/07/20/1555412015595924.full>.

GostкowsкA, Kaja (2013): Wewnątrz- i zewnątrzjęzykowe mechanizmy terminotwórcze. Na przykładzie francuskiej i polskiej terminologii z zakresu inżynierii biomedycznej. Thèse de doctorat. Wrocław: Uniwersytet Wrocławski.

KALISzewsKa, Iwona (2009): Wspomagana komputerowo analiza wywiadów etnograficznych i tekstów folkloru. In: Gabriela GAŃczArczyk et Piotr Grochowski, dir. Folklor w dobie Internetu. Toruń: Wydawnictwo Naukowe Uniwersytetu Mikołaja Kopernika, 197-203.

KuźNik, Anna (2011): Puestos de trabajo híbridos. Cuatro indicadores del carácter heterogéneo de los puestos de trabajo internos en traducción. Sendebar. 22:283-307. Page consultée le 23 juillet 2015, <http://revistaseug.ugr.es/index.php/sendebar/article/view/355/387>.

Kuźnik, Anna (2010/2012): El contenido de los puestos de trabajo de los traductores. El caso de los traductores internos en las empresas de traducción de Barcelona. Thèse de doctorat. Bellaterra. Universitat Autònoma de Barcelona 2010. 582 p. Page consultée le 23 juillet 2015, $<$ http://tdx.cat/handle/10803/5279> (publication 2012. Saarbrücken: AV Akademikerverlag $\mathrm{GmbH} \&$ Co. KG/ Editorial Académica Española. 620 p.).

KuźnıK, Anna (2014a): Praktyki zawodowe jako źródło informacji o rynku pracy i usług z zakresu tłumaczeń prawniczych w Barcelonie. Rocznik Przekładoznawczy. Studia nad teoria, praktyką i dydaktyka przekładu. 9:303-315. Page consultée le 23 juillet 2015, <http:// apcz.pl/czasopisma/index.php/RP/article/view/RP.2014.021/4770>.

KuźNiK, Anna (2014b): Translation as a Paradigmatic Universal, Post-Industrial, KnowledgeBased and Innovative Service, inTRAlinea. online translation journal. special issue Maria 
Piotrowska et Sergiy Tyupa, dir. Challenges in Translation Pedagogy. 13 p. Page consultée le 23 juillet 2015, <www.intralinea.org/specials/article/2098>.

KuźNik, Anna (2015): Étudiants polonais de langue et civilisation françaises et leur avenir professionnel. In: Elżbieta SкiвıŃska, Regina Solová et Kaja Gostкowska, dir. Vingt cinq ans après... Traduire dans une Europe en reconfiguration. Paris: Orizons, 289-309.

KUŹNIK, Anna (2016): Work content of in-house translators in small and medium-sized industrial enterprises. Observing real work situation. JoSTrans. The Journal of Specialised Translation. 25(1): 213-231. Consulté le 14 mai 2016, <www.jostrans.org/issue25/art_kuznik.pdf>.

KuŹNik, Anna (sous presse): Expérience d'un travail coopératif institutionnel avec les acteurs économiques du marché. Projet pilote de traduction à l'Institut d'études romanes de l'Université de Wrocław. In: Enrico Monti et Peter Schnyder, dir. Traduire à plusieurs. Paris: Orizons.

KuŹnik, Anna et Bestué, Carme (2016): Perfiles de prácticas y solución de conflictos emergentes: estudio de un caso de prácticas de traducción jurídica. Estudios Hispánicos. XXIV:93-105.

Kuźnik, Anna et Verd, Joan Miquel (2010): Investigating Real Work Situations in Translation Agencies. Work Content and its Components. Hermes - Journal of Language and Communication Studies, 44:25-43. Page consultée le 23 juillet 2015, <http://download2.hermes.asb. $\mathrm{dk} /$ archive/download/Hermes-44-kuznik\&verd.pdf>.

KuźNiK, Anna, Verd, Joan Miquel et Olalla-Soler, Christian (2016) : Mixed methods, mixed tools. The use of computer software for integrated qualitative and quantitative analysis. Journal of Research Design and Statistics in Linguistics and Communication Science [JRDS], 3(1):76-109. Page consultée le 22 mars 2017, <https://journals.equinoxpub.com/index.php/ JRDS/article/view/32360>.

KwiAteK, Ewelina et Zabrocka, Monika (2014): EGPS - nowe możliwości na drodze kształcenia przyszłych tłumaczy (sprawozdanie). Rocznik Przekładoznawczy. Studia nad teoria, praktyka i dydaktyka przekładu. 9:351-354.

López-Sintas, Jordi, Rojas de Francisco, Laura et García-Állvarez, Ercilia (2015): The Nature of Leisure Revisited: An Interpretation of Digital Leisure. Journal of Leisure Research. 47(1):79-101.

Marco Borillo, Josep Manuel (2016): On the margins of the profession: the work placement as a site for the literary translator trainee's legitimate peripheral participation. The Interpreter and TranslatorTrainer. Published online 29 Apr 2016: 15 p. Page consultée le 10 mai 2016, <http://dx.doi.org/10.1080/1750399X.2016.1154341>.

Planas, Jordi (2011): La relación entre educación y empleo en Europa. Papers. 96(4):1047-1073.

Pym, Anthony, González Núñez, Gabriel, Miquel-Iriarte, Marta, Ramos Pinto, Sara, teixeira, Carlos S. C. et Tesseur, Wine (2014): «Work placements in doctoral research training in the humanities: Eight cases from translation studies». Across Languages and Cultures. 15(1):1-23.

Rojo, Ana (2013): Diseños y métodos de investigación en traducción. Madrid: Editorial Síntesis.

SaWICKI, Piotr (1995): Z romanistycznego sztambucha. In: Teresa Kulak et Wojciech WrZesiński, dir. Studia i materiały z dziejów Uniwersytetu Wrocławskiego, Vol. 4. Wrocław: Wydawnictwo Uniwersytetu Wrocławskiego, 219-249.

SKIBIŃSKA, Elżbieta (2002a): Ewolucja polskiego modelu oferty pracy po roku 1989. In: Grzegorz SzPILA, dir. Język trzeciego tysiąclecia II : zbiór referatów z konferencji, Kraków, 28 lutego-2 marca 2002. Vol. 1. Nowe oblicza komunikacji we współczesnej polszczyźnie. Cracovie: Wydawnictwo Tertium, 203-210.

SкIBIŃsKa, Elżbieta (2002b): Offre d'emploi: modèles français et polonais. Une étude contrastive. Romanica Wratislaviensia. XLIX:42-73.

Sкıвı́́sкA, Elżbieta (2002c): Heurs et malheurs de l'utilisateur des corpus bilingues françaispolonais en classe de traduction. In: Daniel GouAdec, dir. Traduction, terminologie, rédaction. Actes des universités d'été et d'automne 2001 et du colloque international sur la traduction spécialisée, Université de Rennes 2. Paris: La Maison du Dictionnaire, 111-117. 
SKibiŃsKa, Elżbieta (2007): Entre rêve et réalité: insertion professionnelle des diplômés en études françaises, filière Traduction. In: Daniel GouAdec, dir. Traduction, terminologie, rédaction. Actes des universités d'été et d'automne 2006. Actes du colloque international, septembre 2006: Traduction spécialisée. Quelle qualification universitaire pour les traducteurs? Paris: La Maison du Dictionnaire, 87-94.

Skibińska, Elżbieta (2011): Les études romanes en Pologne: la traduction dans la recherche et dans l'enseignement. In: Teresa Giermak-Zielińska, Zbigniew Naliwajek et Joanna Żurowska, dir. Les études romanes / françaises hier et aujourd'hui. 90 ans des études romanes à l'Université de Varsovie. Varsovie: Wydawnictwo Uniwersytetu Warszawskiego, 79-86.

Skibińska, Elżbieta et Kaufman, Stefan (2000): Intégration de la formation des traducteurs dans le cursus philologique: une greffe réussie. Daniel GouAdEc, dir. Formation des traducteurs. Actes du Colloque International, Rennes 2 (24-25 septembre 1999). Paris: La Maison du Dictionnaire, 239-246.

Torres-Hostench, Olga (2010): An overview of the research on occupational integration in translation studies according to a map of occupational integration concepts. Perspectives: Studies in Translatology. 18(1):23-38.

Torres-Hostench, Olga (2012): Occupational integration training in Translation. Meta: Translators' Journal. 57(3):787-812.

UWr Uniwersytet WrocŁaWski $(2011,2012,2013)$ : Liczba studentów wg kierunku i rodzaju studiów (stan na 30/11/2011, 30/11/2012, 30/11/2013). Biuletyn Informacji Publicznej (BIP). Wrocław: Uniwersytet Wrocławski. 1 p., 2 p., 2 p. Page consultée le 23 juillet 2015, <http://bip. uni.wroc.pl/280/183/studenci-uniwersytetu-wroclawskiego-wg-wydzialow-i-trybu-studiow. html>.

Yin, Robert K. (1994): Case study research. Design and Methods. Thousand Oaks, CA: Sage.

\section{ANNEXE 1}

TABLEAU 3

Domaines de l'activité économique et activités effectuées pendant les stages dans les entités d'accueil à haut potentiel d'embauche en Pologne

\begin{tabular}{|c|c|c|c|}
\hline $\begin{array}{l}\text { Domaine d'activité } \\
\text { économique } \\
\text { (nombre d'étudiants } \\
\text { par domaine) }\end{array}$ & $\begin{array}{l}\text { Nom abrégé de l'entité } \\
\text { (nombre d'étudiants } \\
\text { accueillis) }\end{array}$ & Activités effectuées par les étudiants & $\begin{array}{l}\text { Langues } \\
\text { mentionnées }\end{array}$ \\
\hline \multirow[t]{2}{*}{ Culture (2) } & $\begin{array}{l}\text { Dolnośląska Biblioteka } \\
\text { Publiczna, Biblioteka } \\
\text { Romańska (1) }\end{array}$ & $\begin{array}{l}\text { Service aux usagers de la bibliothèque; } \\
\text { gestion des ressources documentaires; } \\
\text { organisation d'événements culturels }\end{array}$ & FR \\
\hline & $\begin{array}{l}\text { Alliance Française; } \\
\text { Stowarzyszenie } \\
\text { Wrocław (1) }\end{array}$ & $\begin{array}{l}\text { Organisation d'événements culturels; } \\
\text { traductions pour des événements } \\
\text { culturels }\end{array}$ & FR \\
\hline \multirow[t]{5}{*}{ Éducation (7) } & El Sueño (1) & $\begin{array}{l}\text { Cours de langues, gestion des cours de } \\
\text { langues et des matériaux didactiques }\end{array}$ & ES \\
\hline & $\begin{array}{l}\text { El Universo / Przystek } \\
\text { Edu (2) }\end{array}$ & $\begin{array}{l}\text { Gestion des cours de langues et des } \\
\text { matériels didactiques }\end{array}$ & ES, EN \\
\hline & Le Rêve (2) & $\begin{array}{l}\text { Préparation des matériaux didactiques } \\
\text { (niveau débutant); cours de langues }\end{array}$ & FR \\
\hline & Kokosowa Wyspa (1) & $\begin{array}{l}\text { Cours de langues (niveau école } \\
\text { maternelle) }\end{array}$ & FR \\
\hline & Przedszkole Nr 147 (1) & $\begin{array}{l}\text { Activités didactiques de musique, langue } \\
\text { et culture françaises (niveau école } \\
\text { maternelle) }\end{array}$ & FR \\
\hline
\end{tabular}




\begin{tabular}{|c|c|c|c|}
\hline \multirow[t]{3}{*}{$\begin{array}{l}\text { Gestion comptable } \\
\text { et financière ( } 3 \text { ) }\end{array}$} & $\begin{array}{l}\text { Biuro Alfa-Omega. } \\
\text { Doradca podatkowy (1) }\end{array}$ & $\begin{array}{l}\text { Traitement des données comptables avec } \\
\text { l'utilisation des logiciels; gestion des } \\
\text { factures des clients francophones; } \\
\text { fiscalité }\end{array}$ & FR \\
\hline & $\begin{array}{l}\text { Ernst \& Young Global } \\
\text { Services Poland (1) }\end{array}$ & $\begin{array}{l}\text { Comptabilité, externalisation des } \\
\text { processus d'affaires (en EN: BPO), audit } \\
\text { de la documentation comptable relative } \\
\text { au détachement des clients étrangers, } \\
\text { service à la clientèle francophone, } \\
\text { préparation des paies et autres } \\
\text { opérations propres aux systèmes } \\
\text { comptables }\end{array}$ & FR, EN \\
\hline & Deutsche Bank (1) & $\begin{array}{l}\text { Service aux clients francophones, } \\
\text { préparation des matériaux informatifs et } \\
\text { publicitaires concernant les produits } \\
\text { financiers de la banque }\end{array}$ & FR \\
\hline $\begin{array}{l}\text { Gestion des } \\
\text { ressources } \\
\text { humaines (1) }\end{array}$ & $\begin{array}{l}\text { AP Uniapol } \\
\text { Developement (1) }\end{array}$ & $\begin{array}{l}\text { Recrutement et sélection des candidats } \\
\text { pour les départements francophones des } \\
\text { entreprises, principalement du domaine } \\
\text { de bâtiment, préparation des profils des } \\
\text { candidats, tâches administratives }\end{array}$ & FR \\
\hline \multirow[t]{2}{*}{$\begin{array}{l}\text { Gestion ventes et } \\
\text { marketing (2) }\end{array}$} & $\begin{array}{l}\text { Euro Pro Doradztwo } \\
\text { Biznesowe (1) }\end{array}$ & $\begin{array}{l}\text { Service aux clients internationaux, } \\
\text { préparation des matériaux informatifs et } \\
\text { publicitaires, traductions dans le } \\
\text { domaine de soutien de marketing aux } \\
\text { PME et vente des caméras thermiques }\end{array}$ & FR, EN \\
\hline & CeDo (1) & $\begin{array}{l}\text { Service aux clients internationaux, } \\
\text { gestion des ventes de vêtements et } \\
\text { accessoires }\end{array}$ & FR, EN \\
\hline \multirow[t]{3}{*}{$\begin{array}{l}\text { Publicité (arts } \\
\text { visuels numériques) } \\
\text { (3) }\end{array}$} & $\begin{array}{l}\text { Agencja Reklamowa } \\
\text { Neck (1) }\end{array}$ & $\begin{array}{l}\text { Service aux clients francophones, } \\
\text { marketing: recherche de clients } \\
\text { potentiels des pays francophones }\end{array}$ & FR \\
\hline & Kreatywni24 (1) & $\begin{array}{l}\text { Service aux clients francophones, gestion } \\
\text { de l'entreprise, préparation des } \\
\text { matériaux informatifs et publicitaires, } \\
\text { traductions }\end{array}$ & FR, ES \\
\hline & Artgeist (1) & $\begin{array}{l}\text { Service aux clients francophones, } \\
\text { traductions pour la production et vente } \\
\text { de tableaux contemporains (numérisés } \\
\text { ou non) }\end{array}$ & FR \\
\hline \multirow{3}{*}{$\begin{array}{l}\text { Télécommunication, } \\
\text { internet, produits } \\
\text { multimédias (IT, } \\
\text { ICT) (4) }\end{array}$} & HCL Poland (1) & $\begin{array}{l}\text { Service aux clients francophones du } \\
\text { groupe Volvo, domaine informatique }\end{array}$ & FR \\
\hline & $\begin{array}{l}\text { Telecommunications } \\
\text { Operations \& Service } \\
\text { Centre (ACN) (2) }\end{array}$ & $\begin{array}{l}\text { Service aux clients francophones, service } \\
\text { comptabilité et site web avec FAQ }\end{array}$ & FR, EN \\
\hline & OpsTalent (1) & $\begin{array}{l}\text { Service technique aux clients } \\
\text { internationaux - usagers des jeux vidéo } \\
\text { de Ubisoft Entertainment, actualisation } \\
\text { du manuel du jeu }\end{array}$ & FR, EN \\
\hline \multirow[t]{3}{*}{ Transport aérien (4) } & $\begin{array}{l}\text { GTL-LOT Usługi } \\
\text { Lotniskowe (1) }\end{array}$ & $\begin{array}{l}\text { Service aux clients internationaux dans } \\
\text { l'aéroport, analyse comparative des } \\
\text { procédures de différentes lignes } \\
\text { aériennes concernant les bagages, } \\
\text { contrôle des bagages des passagers }\end{array}$ & FR, EN \\
\hline & Qatar Airways (1) & $\begin{array}{l}\text { Service aux clients francophones dans le } \\
\text { domaine de l'aviation et du transport } \\
\text { aérien }\end{array}$ & FR \\
\hline & SkyTaxi (2) & $\begin{array}{l}\text { Service aux clients francophones, } \\
\text { préparation des matériaux informatifs, } \\
\text { traductions }\end{array}$ & FR \\
\hline
\end{tabular}




\begin{tabular}{|c|c|c|c|}
\hline \multirow[t]{2}{*}{$\begin{array}{l}\text { Hôtellerie } \\
\text { (tourisme) (2) }\end{array}$} & Corner Hostel (1) & $\begin{array}{l}\text { Service aux clients internationaux à la } \\
\text { réception, tâches d'assistance aux } \\
\text { chambres et de gastronomie à l'hôtel, } \\
\text { information touristique }\end{array}$ & $\begin{array}{l}\text { EN, FR, ES, } \\
\text { GE }\end{array}$ \\
\hline & Grampa's Hostel (1) & $\begin{array}{l}\text { Service aux clients internationaux à la } \\
\text { réception, tâches d'assistance aux } \\
\text { chambres et de gastronomie à l'hôtel, } \\
\text { information touristique }\end{array}$ & $\mathrm{FR}, \mathrm{EN}$ \\
\hline \multirow[t]{2}{*}{$\begin{array}{l}\text { Restauration } \\
\text { (culture, tourisme) } \\
\text { (2) }\end{array}$} & Le Bistrot Parisien (1) & $\begin{array}{l}\text { Serveuse et consultante culturelle, } \\
\text { service aux clients internationaux, } \\
\text { traductions, organisation d'activités de } \\
\text { promotion }\end{array}$ & FR, EN, ES \\
\hline & Central Bar 49 (1) & $\begin{array}{l}\text { Services aux clients internationaux, } \\
\text { gestion des fournisseurs, comptabilité, } \\
\text { activités de marketing }\end{array}$ & FR, EN, IT \\
\hline \multirow[t]{6}{*}{ Traduction (7) } & Biuro tłumaczeń (1) & $\begin{array}{l}\text { Traduction des documents, préparation } \\
\text { des traductions assermentées }\end{array}$ & FR \\
\hline & $\begin{array}{l}\text { Biuro tłumacza } \\
\text { przysięgłego (język } \\
\text { francuski i włoski) (2) }\end{array}$ & $\begin{array}{l}\text { Traduction des documents, préparation } \\
\text { des traductions assermentées, } \\
\text { traductions juridiques et économiques, } \\
\text { traduction de la correspondance privée, } \\
\text { conversations téléphoniques }\end{array}$ & FR \\
\hline & $\begin{array}{l}\text { Biuro tłumacza } \\
\text { przysięgłego (język } \\
\text { francuski) (1) }\end{array}$ & $\begin{array}{l}\text { Traduction des documents, préparation } \\
\text { des traductions assermentées, } \\
\text { traductions juridiques et techniques, } \\
\text { interprétariat }\end{array}$ & FR \\
\hline & $\begin{array}{l}\text { Twój Tłumacz } \\
\text { (TwojTlumacz.pl) (1) }\end{array}$ & $\begin{array}{l}\text { Service aux clients, traductions, } \\
\text { élaboration des glossaires } \\
\text { terminologiques }\end{array}$ & FR, EN, GE \\
\hline & $\begin{array}{l}\text { Uniwersytet } \\
\text { Warmińsko-Mazurski; } \\
\text { Instytut } \\
\text { Słowiańszczyzny } \\
\text { Wschodniej (1) }\end{array}$ & $\begin{array}{l}\text { Traductions liées à l'organisation } \\
\text { d'événements et préparation des projets } \\
\text { culturels, didactiques et scientifiques } \\
\text { dans un établissement d'enseignement } \\
\text { supérieur }\end{array}$ & FR, EN \\
\hline & $\begin{array}{l}\text { Laboratorium } \\
\text { Analityczne Diagnoza } \\
\text { (1) }\end{array}$ & $\begin{array}{l}\text { Traductions et interprétation, } \\
\text { communication avec le fournisseur et } \\
\text { gestion de l'achat d'équipement pour } \\
\text { l'analyse médicale }\end{array}$ & FR \\
\hline $\begin{array}{l}\text { Soutien juridique } \\
\text { (documentation) (1) }\end{array}$ & $\begin{array}{l}\text { Kancelaria Adwokacka } \\
\text { (1) }\end{array}$ & $\begin{array}{l}\text { Gestion de l'agenda, service aux clients, } \\
\text { documentation et préparation des écrits, } \\
\text { traductions }\end{array}$ & FR \\
\hline
\end{tabular}

Légende: FR - français, ES - espagnol, IT - italien, EN - anglais, GE - allemand 